\title{
Consistency results in topology and homotopy theory
}

\section{Jaykov Foukzon}

Israel Institute of Technology, Department of Mathematics, Haifa, Israel

\section{Email address:}

jaykovfoukzon@list.ru

\section{To cite this article:}

Jaykov Foukzon. Consistency Results in Topology and Homotopy Theory. Pure and Applied Mathematics Journal. Special Issue: Modern Combinatorial Set Theory and Large Cardinal Properties. Vol. 4, No. 1-1, 2015, pp. 1-5. doi: 10.11648/j.pamj.s.2015040101.11

\begin{abstract}
Main results is: (1) let $\kappa$ be an inaccessible cardinal and $H_{k}$ is a set of all sets having hereditary size less then $\kappa$, then $\operatorname{Con}\left(Z F C+\left(V=H_{k}\right)\right),(2)$ there is a Lindelöf $T_{3}$ indestructible space of pseudocharacter $\leqslant N_{l}$ and size $N_{2}$ in $L$.
\end{abstract}

Keywords: Inner Model of $Z F C$, Inaccessible Cardinal, Weakly Compact Cardinal, Lindelöf Space, Indestructible Space, $N_{l}$ Borel Conjecture

\section{Introduction}

A note on the term large cardinal is in order. A cardinal number is 'large' if the assumption of its existence, when added to the axioms of $Z F C$, proves the consistency of $Z F C$. This works as follows, for any cardinal $\kappa$ one can consider the set $H_{\kappa}$ - the set of all sets which have size less than $\kappa$ and whose members and members of members and . . . all have size less than $\kappa$. Loosely speaking $\kappa$ is large if $H_{\kappa}$ is a model of $Z F C$.

Theorem 1. [7]. $\neg \operatorname{Con}\left(Z F C+\left(V=H_{\kappa}\right)\right)$.

A cardinal number $\kappa$ is an inaccessible cardinal (also a strongly inaccessible cardinal) if it is regular and $2^{\lambda}<\kappa$ whenever $\lambda<\kappa$ is a cardinal. $\kappa$ is a weakly compact cardinal if it is inaccessible and, whenever $T$ is a tree of height $\kappa$ with levels of size less than $\kappa$, then $T$ has a branch of length $\kappa . \kappa$ is a measurable cardinal if there is a nonprincipal $\kappa$-complete ultrafilter (i.e., closed under intersections of size less than $\kappa$ ). A cardinal $\kappa$ is a strongly compact cardinal if every $\kappa$ complete filter can be extended to a $\kappa$-complete ultrafilter. All of these cardinals have several equivalent formulations. The easiest to state are often in terms of ultrafilters, but the most useful involve elementary embeddings.

Definition 1. Any inner model of $Z F C$ is a class $M=$ $\{x: \phi(x)\}$, for some formula $\phi$, such that $Z F C$ holds in $M$. An elementary embedding $j: V \rightarrow M$, where $V$ is the universe of sets, is a function such that for every $a_{1}, \ldots, a_{n} \in$ $V$, and for every formula $\psi\left(x_{1}, \ldots, x_{n}\right), \psi\left(a_{1}, \ldots, a_{n}\right)$ holds if and only if $\psi\left(j\left(a_{1}\right), \ldots, j\left(a_{n}\right)\right)$ holds in $M . M$ is closed under $\lambda$-sequences If ${ }^{\lambda} M$, the class of all $\lambda$-sequences of members of $M$, is a subclass of $M$.

From definitions one can prove that.
Theorem 2. $\kappa$ is measurable if and only if there is an inner model $M$ closed under $\kappa$-sequences and an elementary embedding $j: V \rightarrow M$ such that $j(\kappa)>\kappa$.

From Theorem 1 and Theorem 2 one obtain directly.

Theorem 3. Assume that $\kappa$ is measurable cardinal. There is no any inner model $M$ of $Z F C$ such mentioned above.

Large cardinals sometimes appear in purely topological contest. Well known from Jones' Lemma that $2^{|D|} \leq 2^{d(X)}$ whenever $D$ is a closed discrete subset of a normal space $X$, where $d(X)$ denotes the density of $X$. The extent of $X$, denoted $e(X)$, is the supremum of the cardinalities of the closed discrete subsets of $X$ and this suggests the natural question whether also $2^{e(X)} \leq 2^{d(X)}$ for normal spaces. This leads to inaccessible cardinals: if $2^{e(X)}>2^{d(X)}$ then $e(X)$ is a weakly inaccessible cardinal. For example from an inaccessible cardinal one can prove the consistency of the existence of a normal space satisfying the above inequality. Strongly compact and weakly compact cardinals can be equivalently formulated topologically: $\kappa$ is strongly compact if and only if the $\kappa$-box product of $\kappa$-compact spaces is $\kappa$ compact, wherein one takes the Tychonoff Product Theorem and replaces "finite" by " $<\kappa$ " everywhere; $\kappa$ is weakly compact is the ordinary product of $\kappa$-compact spaces is again $\kappa$-compact. The most significant uses of large cardinals in topology occur in contexts in which one is proving the consistency of universal statements about objects of unbounded cardinality, for example, the Normal Moore Space Conjecture: all normal Moore spaces are metrizable, or the Moore-Mrówka problem: compact spaces of countable tightness are sequential. 
The latter is an application of the Proper Forcing Axiom (PFA) [33], which is proved consistent from the consistency of a supercompact cardinal, and applications of which - in contrast to those of Martin's Axiom- often require the practitioner to actually do some forcing. Frequently, finer analyses of PFA consequences reveal that in fact one need only consider objects of bounded cardinality, in particular $\aleph_{1}$. In such cases, a more delicate forcing argument enables one to avoid large cardinals. That is the case with the MooreMrówka problem referred to above.

\section{Consistency Results in Topology}

Definition 1. [28].A Lindelöf space is indestructible if it remains Lindelöf after forcing with any countably

closed partial order.

Theorem 1.[29].If it is consistent with ZFC that there is an inaccessible cardinal, then it is consistent with

ZFC that every Lindelöf $T_{3}$ indestructible space of weight $\leq \aleph_{1}$ has size $\leq \aleph_{1}$.

Corollary 1.[29] The existence of an inaccessible cardinal and the statement: $\mathcal{L}\left[T_{3}, \leq \aleph_{1}, \leq \aleph_{1}\right] \triangleq$ "every

Lindelöf $T_{3}$ indestructible space of weight $\leq \aleph_{1}$ has size $\leq \aleph_{1}$ ", are equiconsistent.

Theorem 2. $\neg \operatorname{Con}\left(Z F C+\mathcal{L}\left[T_{3}, \leq \aleph_{1}, \leq \aleph_{1}\right]\right)$.

Proof. Theorem 1 immediately follows from Theorem 3.6 [32] and Corollary 1.

Definition 2. The $\aleph_{1}$-Borel Conjecture is the statement: $B C\left[\aleph_{1}\right] \triangleq$ "a Lindelöf space is indestructible if and only

if all of its continuous images in $[0 ; 1]^{\omega_{1}}$ have cardinality $\leq \kappa_{1}^{\prime \prime}$.

Theorem 3. [29]. If it is consistent with ZFC that there is an inaccessible cardinal, then it is consistent with ZFC

that the $\aleph_{1}$-Borel Conjecture holds.

Corollary 2. The $\aleph_{1}$-Borel Conjecture and the existence of an inaccessible cardinal are equiconsistent.

Theorem 4. $\neg \operatorname{Con}\left(Z F C+B C\left[\aleph_{1}\right]\right)$.

Proof. Theorem 4 immediately follows from Theorem 3.6 [32] and Corollary 2.

Theorem 5.[29]. If $\omega_{2}$ is not weakly compact in $L$, then there is a Lindelöf $T_{3}$ indestructible space of

pseudocharacter $\leq \aleph_{1}$ and size $\aleph_{2}$.

Corollary 3.The existence of a weakly compact cardinal and the statement: $\mathcal{L}\left[T_{3}, \leq \aleph_{1}, \leq \aleph_{2}\right] \triangleq$ "there is no

Lindelöf $T_{3}$ indestructible space of pseudocharacter $\leq \aleph_{1}$ and size $\aleph_{2}$ are equiconsistent.

Theorem 6.There is a Lindelöf $T_{3}$ indestructible space of pseudocharacter $\leq \aleph_{1}$ and size $\aleph_{2}$ in $L$.

Proof.Theorem 6 immediately follows from Theorem 3.6 [32] and Theorem 5.

Theorem 7. $\neg \operatorname{Con}\left(Z F C+\mathcal{L}\left[T_{3}, \leq \aleph_{1}, \leq \aleph_{2}\right]\right)$.

Theorem 8. (Solovay) If $\omega_{2}$ is not inaccessible in $L$, then there is a Kurepa tree.

Corollary 4. (Solovay) There is a Kurepa tree in $L$.

Proof. Immediately follows from Theorem 3.6 [32] and Theorem 5.

\section{Consistency Results in Homotopy Theory}

Classical homotopy idempotent functors appear frequently in algebraic topology. A homotopy idempotent functor is a functor $E$ from some model category to itself that carries weak equivalences to weak equivalences and is equipped with a natural transformation [36]: $\delta:$ Id $\rightarrow E$ such that both $\delta E$ and $E \delta$ induce weak equivalences $E X \cong E E X$ for all $X$. In [37], Farjoun developed a theory of localization with respect to any map $f: A \rightarrow B$. Farjoun's construction associates functorially with each space $X$ a map $X \rightarrow L_{f} X$ which is universal, up to homotopy, among maps from $X$ into fibrant spaces $Y$ such that the map off function complexes

$$
\operatorname{map}(B, Y) \rightarrow \operatorname{map}(A, Y)
$$

induced by $f$ is a weak equivalence. We assume that any spaces will be simplicial sets, and maps and function complexes will be unbased. For each map $f$, the functor $L_{f} X$ is homotopy idempotent and continuous, that is, it induces a natural map of function complexes

$$
\operatorname{map}(X, Y) \rightarrow \operatorname{map}\left(L_{f} X, L_{f} Y\right)
$$

for all $X$ and $Y$, preserving composition and identity. Dror Farjoun to ask in his paper [37]: if every homotopy idempotent functor on simplicial sets is equivalent to some $f$-localization?. In [35] was shown that it is impossible to answer this question "yes" using only $Z F C$ axioms.

Moreover, a negative answer to this question in $Z F C$ is not to be expected, as it would imply the inconsistency of certain large-cardinal axioms that are believed to be consistent with $Z F C$ after many years of related developments in set theory.

We remind that an cardinal $\lambda$ is regular if it is infinite and cannot be expressed as a sum of cardinals $\sum_{i<\alpha} \lambda_{i}$, where $\alpha<\lambda, \lambda_{i}<\alpha$ for all $i$. Otherwise, $\lambda$ is called singular.

Definition 1. A partially ordered set is called directed if every pair of elements has an upper bound. More generally, for any regular cardinal $\lambda$, a partially ordered set is called $\lambda$ directed if every subset of cardinality smaller than $\lambda$ has an upper bound.

Definition 2. An object $X$ of a category $C$ is called $\lambda$ presentable, where $\lambda$ is a regular cardinal, if

the functor $C(X,-)$ preserves $\lambda$-directed colimits, that is, colimits of diagrams $D: I \rightarrow C$ where $I$ is a $\lambda$-directed partially ordered set.

Definition 3. A category $C$ is locally presentable if it is cocomplete and there is a regular cardinal $\lambda$ and a set $X$ of $\lambda$ presentable objects such that every object of $C$ is a $\lambda-$ directed colimit of objects from $X$.

Definition 4. An idempotent monad on a category $C$ is a pair $(E, \delta)$ consisting of a functor $E$ and a natural transformation $\delta:$ Id $\rightarrow E$ such that $\delta_{E X}: E X \rightarrow E E X$ is an isomorphism for every object $X$, and $\delta_{E X}=E \delta_{X}$ for all $X$.

Definition 5. For simplicity, we say that a functor $E$ is idempotent if it is part of an idempotent monad. Then we also call it a reflection or a localization.

Definition 6. An object $X$ and a morphism $f: A \rightarrow B$ in a 
category $C$ are orthogonal if the map

$$
C(f, X): C(B, X) \rightarrow C(A, X)
$$

is bijective; that is, $X$ and $f$ are orthogonal iff or every morphism $g: A \rightarrow X$ there is a unique morphism $h: B \rightarrow X$ such that $h^{\circ} f=g$. The class ofobjects that are orthogonal to a given class $S$ of morphisms is denoted by $S^{\perp}$ and called the orthogonal complement of $S$. The same notation is used by exchanging the role ofobjects and morphisms.

Definition 7. A class $D$ ofobjects in a category $C$ is called reflective if it is the class of $E$ local objects for some idempotent functor $E$. (No distinction is made here between

a class of objects and the full subcategory with those objects.)

Definition 8. A class of objects $D$ is called a smallorthogonality class if there is a set $M$ of morphisms (not a proper class) such that $M^{\perp}=D$.

Definition 9. A functor $E$ on the category of simplicial sets is homotopy idempotent if it carries weak equivalences to weak equivalences and is equipped with a natural transformation: $\delta$ : Id $\rightarrow E$ such that $\delta_{E X} \cong E \delta_{X}$ and $\delta_{E X}: E X \rightarrow E E X$ is a weak equivalence for all $X$.

It well known that, in the category of groups, for every (possibly proper) class $S$ of epimorphisms, the orthogonal complement $S^{\perp}$ is reflective [38].

Definition 10. For any group $G$, let $T G$ be the intersection of all kernels of epimorphisms from $G$ onto groups in $S^{\perp}$.

Then $E G=G / T G$ is the desired reflection.

Definition11.In the special case when $S$ is a class of homomorphisms of the form $A_{\alpha} \rightarrow 0$, where $A_{\alpha}$ ranges over a set or a class $A$ of groups, the corresponding reflection will be called $A$-reduction and denoted $P_{A}$.

Thus, a group $G$ is $A$-reduced if and only if the set $\operatorname{Hom}\left(A_{\alpha}, G\right)$ is trivial for every $A_{\alpha}$ in $A$.

For each cardinal $\kappa$, we denote by $Z^{\kappa}$ the cartesian product group of $\kappa$ copies of the additive group of integers; that is, $Z^{\kappa}$ is the abelian group of all functions $f \kappa: \rightarrow Z$. For a function $f \in Z^{\kappa}$, the support supp $(f)$ is the set of indices $i \in \kappa$ for which $f(i) \neq 0$.

We write $Z^{<\kappa}$ to designate the set of all functions $\mathrm{f} \in Z^{\kappa}$ such that the cardinality of $\operatorname{supp}(f)$ is smaller than $\kappa$.

Theorem1.[35] Suppose that all cardinals are nonmeasurable. If $\mathrm{A}$ is the class of groups $Z^{\kappa} / Z^{<\kappa}$ for all cardinals $\kappa$, then there is no single group homomorphism $\varphi$ such that $\varphi$-localization is isomorphic to A-reduction on the category of groups.

We remind that an uncountable cardinal $\mu$ is measurable if it admits a nontrivial, two valued, $\mu$-additive measure, that is, if a function $\mu$ can be defined on any set X of cardinality $\mu$ assigning to each subset of $\mathrm{X}$ a value 0 or 1 , in such a way that $\mu(\mathrm{X})=1, \mu(\mathrm{x})=0$ for all $x \in X$, and $\mu\left(\mathrm{U}_{\mathrm{i}} \mathrm{A}_{\mathrm{i}}\right)=$ $\sum_{i} \mu\left(A_{i}\right)$ fthe subsets $A_{i}$ are pairwise disjoint and the set ofindices i has cardinality smaller than $\mu$.

Theorem 2. If $\mathrm{A}$ is the class of groups $Z^{\kappa} / Z^{<\kappa}$ for all cardinals $\kappa$, then there is no single group homomorphism $\varphi$ such that $\varphi$-localization is isomorphic to A-reduction on the category of groups.

Proof. Note that the existence of measurable cardinals cannot be proved in $Z F C$, since every measurable cardinal is strongly inaccessible; see [39].Therefore Theorem2 immediately follows from Theorem 3.6 [32] and Theorem 1.

Theorem3.[35]. Suppose that all cardinals are nonmeasurable. Then there is a homotopy idempotent functor $E$ on simplicial sets that is not equivalent to $f$-localization for any map $f$.

Theorem4. There is a homotopy idempotent functor $E$ on simplicial sets that is not equivalent to $f$-localization for any $\operatorname{map} f$.

Proof. Note that the existence of measurable cardinals cannot be proved in $Z F C$, since every measurable cardinal is strongly inaccessible; see [39].Therefore Theorem4 immediately follows from Theorem 3.6 [32] and Theorem3. Definition 11. A class of objects is called rigid if it admits no other morphisms than identities.

Vopenka's principle [39]: no locally presentable category contains a rigid proper class of objects.

Definition 12. Recall that, if $E$ is a homotopy idempotent functor on simplicial sets, a simplicial set $X$ is called $E$ acyclic if $E X$ is contractible. A universal E-acyclic space is a simplicial set $U$ such that the nullification $P U$ kills the same simplicial sets as $E$ does.

Theorem 5. [35] The existence of a universal E-acyclic space for every homotopy idempotent functor $\mathrm{E}$ on simplicial sets is ensured by Vopenka's principle. However, if we assume that measurable cardinals do not exist, then there are homotopy idempotent functors on simplicial sets for which no universal acyclic space exists.

Theorem6. There are homotopy idempotent functors on simplicial sets for which no universal acyclic space exists.

Theorem 6 immediately follows from Theorem 3.6 [32] and Theorem5.

\section{Von Neumann's Problem and Large Cardinals}

In 1937 von Neumann asked whether every ccc weakly distributive complete Boolean algebra is a measure algebra [40].

Definition 1. A subset of a Boolean algebra is an antichain if it consists of nonzero elements but the meet of any two of its members is zero.

Definition 2. A Boolean algebra is $c c c$ if it does not have uncountable antichains.

Definition 3. A complete Boolean algebra is weakly distributive if for every sequence $A_{n}(n \in \mathbb{N})$ of maximal antichains there is a maximal antichain $A$ such that for every $a \in A$ and $n \in \mathbb{N}$ the set $\left\{b \in A_{n} \mid b \wedge a \neq 0\right\}$ is finite.

Definition 4. A complete Boolean algebra $B$ is a measure algebra if it carries a $\sigma$-additive measure $\mu: B \rightarrow[0,1]$ that is strictly positive: $\mu(a)=0$ implies $a=0$.

Definition 5. $\varphi: B \rightarrow[0,1]$ is a submeasure if $\varphi(0)=0$ and it is monotonic and subadditive: $\varphi(a \cup b) \leq \varphi$ (a) $+\varphi(b)$.

Definition5. A complete Boolean algebra $B$ is a Maharam algebra if it carries a strictly positive continuous submeasure.

Bellow $o(\kappa)=$ denotes the Mitchell order of ameasurable 
cardinal $\kappa$.

Recall that every measure algebra is a Maharam algebra, and every Maharam algebra has $c c c$ and is weakly distributive

Theorem 1. [41]. Assume every $c c c$ weakly distributive complete Boolean algebra is a Maharam algebra. Then there is an inner model with a measurable cardinal $\kappa$ such that $(\kappa)=\kappa^{++}$.

Theorem 2. There is $c c c$ weakly distributive complete Boolean algebra $B$ such that $B$ is no Maharam algebra.

Theorem 3. [41]. Assume every weakly distributive complete Boolean algebra $B$ such that every completely countably generated subalgebra is a measure algebra and $B$ has property $K$ is a Maharam algebra. Then there is an inner model with a measurable cardinal $\kappa$ such that $(\kappa)=\kappa^{++}$.

Theorem 4. There is a weakly distributive complete Boolean algebra $B$ such that every completely countably generated subalgebra is a measure algebra and $B$ hasproperty $K$ such that $B$ is no Maharam algebra.

Theorem 5. [42] For any infinite regular cardinal $\kappa$, the following are equivalent:

(1) Every $\kappa$-complete filter $F$ on a $<\kappa$-distributive complete Boolean algebra $B$ is contained in a $\kappa$-complete ultrafilter $U \subseteq B$.

(2) $\kappa$ is strongly compact.

Theorem 6. For any infinite regular cardinal $\kappa$ there is $\kappa$ complete filter $F$ on a $<\kappa$-distributive complete Boolean algebra $B$ such that $F$ is not contained in a $\kappa$-complete ultrafilter $U \subseteq B$.

\section{Conclusions}

Whenever if one uses inexistence large cardinals to establish the consistency of an topological statement $\phi$, the canonical way one shows that a statement $\neg \phi$ imply the an existence an large cardinals is to show that if $\neg \phi$ holds, then there is an inner model $M$ which has a large cardinal and so statement $\phi$ holds.For example, note that the consistency of the existence of an inaccessible cardinal enables one to prove the consistency of there is Kurepa trees, one then shows that if there is no Kurepa tree, then $\aleph_{2}$ is an inaccessible cardinal in Gödel's constructible universe $L$ and hence that it is consistent that there is an inaccessible cardinal in $L$ and therefore: there is a Kurepa tree in $L$.

\section{References}

[1] M. H. Löb, Solution of a Problem of Leon Henkin. The Journal of Symbolic Logic 20 (2): 115-118.(1955)

[2] C. Smorynski, Handbook of mathematical logic, Edited by J. Barwise. North-Holland Publishing Company, 1977

[3] T. Drucker,Perspectives on the History of Mathematical Logic, Boston : Birkhauser, 2008.

[4] A. Marcja, C. Toffalori, A guide to classical and modern model theory. Springer, 2003, 371 p. Series: Trends in Logic, Vol.19.
[5] F. W. Lawvere, C. Maurer, G. C. Wraith, Model theory and topoi, ISBN: 978-3-540-07164-8

[6] D. Marker, Model theory: an introduction.(Graduate Texts in Mathematics, Vol. 217). Springer 2002.

[7] J. Foukzon, Generalized Löb's Theorem. http://arxiv.org/abs/1301.5340

[8] J. Foukzon, An posible generalization of the Löb's theorem. AMS Sectional Meeting AMS Special Session.Spring Western Sectional Meeting University of Colorado Boulder, Boulder, CO April 13-14, 2013. Meeting \#1089 http://www.ams.org/amsmtgs/2210_abstracts/1089-03-60.pdf

[9] E. Mendelson, Introduction to Mathematical Logic, Edition: 4th,1997. ISBN-10: 0412808307

[10] U. R. Schmerl, Iterated Reflection Principles and the $\omega-$ Rule.The Journal of Symbolic Logic, Vol. 47, No. 4, pp.721733.

[11] S. Feferman, Transfinite recursive progressions of axiomatic theories. Journal of Symbolic Logic 27:259-316.

[12] S. Feferman,Systems of predicative analysis. Journal of Symbolic Logic 29:1-30.

[13] S. Feferman, Reflecting on incompleteness. Journal of Symbolic Logic 56:1-49.

[14] S. Feferman, C. Spector, Incompleteness along paths in progressions of theories. Journal of Symbolic Logic 27:383-390 .

[15] Kreisel, G. and A. Lévy, 1968. Reflection principles and their use for establishing the complexity of axiomatic systems. Zeitschrift für mathematische Logik und Grundlagen der Mathematik 14:97-142.

[16] P.Lindstrom, "First order predicate logic and generalized quantifiers", Theoria, Vol. 32, No.3. pp. 186-195, December 1966.

[17] F.R. Drake," Set Theory: An Introduction to Large Cardinal", Studies in Logic and the Foundations of Mathematics,V.76. (1974). ISBN 0-444-105-35-2

[18] A. Kanamori, "The Higher Infinite: Large Cardinals in Set Theory from Their Beginnings (2003) Springer. ISBN 3-54000384-3

[19] W.V.O.,Quine, "New Foundation for Mathematical Logic," American Mathematical Monthly, vol. 44, (1937) pp. 70-80.

[20] R.B.Jensen,"On the consistency of a slight (?) modification of Quine's 'New Foundation',"Synthese, vol.19 (1969),250-263.

[21] M.R. Holmes,"The axiom of anti-foundation in Jensen's 'New Foundations with ur-elements,' " Bulletin de la Societe Mathematique de la Belgique, Series B.

[22] M.R. Holmes, "Elementary Set Theory with a Universal Set," vol.10 of Cahiers du Centre de Logique, Universite Catholique de Louvain Lovain-la-Neuve, Belgium, 1998.

[23] M.R. Holmes, "The Usual Model Construction for NFU Preserves Information," Notre Dame J. Formal Logic, Volume 53, Number 4 (2012), 571-580.

[24] A. Enayat, "Automorphisms, Mahlo Cardinals,and NFU," AMS Special Session. Nonstandard models of Arithmetic and Set Theory. January 15-16 2003,Baltimore, Maryland. 
[25] R.M.Solovay, R.D. Arthan, J.Harrison, "The cosistency strengths of NFUB," Front for the arXiv,math/9707207

[26] A. Brovykin, "On order-types of models of arithmetic". Ph.D. thesis pp.109, University of Birmingham 2000. http://logic.pdmi.ras.ru/ andrey/phd.pdf A.Brovykin,"Ordertypes of models of arithmetic and a connection with arithmetic saturation", Lobachevskii J. Math., 2004, Volume 16, Pages 3 $-15$

[27] P.J. Cohen, "Set Theory and the Continuum Hypothesis". ISBN: 9780486469218

[28] F.D. Tall, On the cardinality of Lindelöf spaces with points $G_{\delta}$, Topology and its Applications 63 (1995), 21--38.

[29] R.R. Dias,F. D. Tall, Instituto de Matemática e Estatística Universidade de São Paulo,15-th Galway Topology Colloquium, Oxford, 2012.

[30] J. Foukzon, Strong Reflection Principles and Large Cardinal Axioms. Fall Southeastern Sectional Meeting University of Louisville, Louisville, KY October 5-6, 2013 (Saturday Sunday) Meeting \#1092

[31] J. Foukzon, Generalized Lob's Theorem. Strong Reflection Principles and Large Cardinal Axioms. Consistency Results in Topology,IX Iberoamerican Conference on Topology and its Applications 24-27 June, 2014 Almeria, Spain. Book of abstracts, p.66.

[32] J. Foukzon, Generalized Löb's Theorem. Strong Reflection Principles and Large Cardinal Axioms. Consistency Results in Topology, International Conference on Topology and its Applications, July 3-7, 2014, Nafpaktos, Greece. Book of abstracts, p.81.
[33] J. Foukzon, Generalized Lob's Theorem. Strong Reflection Principles and Large Cardinal Axioms. Consistency Results in Topology. http://arxiv.org/abs/1301.5340

[34] F.D. Tall, Consistency results in topology, II: forcing and large cardinals (extended version), Topology Atlas: http://at.yorku.ca/p/a/a/o/08.htm

[35] C.Casacubertaa, D.Scevenelsb, J. H. Smith, Implications of large-cardinal principles in homotopical localization, Advances in Mathematics 197 (2005) 120 - 139

[36] D.G. Quillen, Homotopical Algebra, Lecture Notes in Mathematics, vol. 43, Springer, Berlin Heidelberg New York, 1967.

[37] E. Dror Farjoun, Cellular Spaces, Null Spaces and Homotopy Localization, Lecture Notes in Math. vol. 1622, SpringerVerlag, Berlin Heidelberg New York, 1996.

[38] C. Casacuberta, J. L. Rodr'iguez, and D. Scevenels, Singly generated radicals associated with varieties of groups, in: Proceedings of Groups St Andrews 1997 in Bath, London Math. Soc. Lecture Note Ser. vol. 260, Cambridge University Press, Cambridge, 1999, 202-210.

[39] T. Jech, Set Theory, Academic Press, New York, 1978.

[40] D. Maharam. Problem 167. In D. Mauldin, editor, The Scottish Book, pages 240-243. Birkhauser, Boston, 1981.

[41] Farah, Ilijas, and B. Veličković. "Von Neumann's Problem and Large Cardinals." Bulletin of the London Mathematical Society 38.6 (2006).

[42] J. D. Hamkins and D. E. Seabold, Well-founded Boolean ultrapowers as large cardinal embeddings. http://arxiv.org/abs/1206.6075v1 